\title{
Prevalence and trends in obesity among Austrian conscripts from 1983 to 2017
}

\author{
Lin Yang · Alfred Juan · Thomas Waldhoer (D)
}

Received: 12 June 2021 / Accepted: 16 August 2021

(C) The Author(s) 2021

\begin{abstract}
Summary This article describes the recent prevalence and trend in weight status in young men over three and half decades among Austrian conscripts overall and by subgroups defined by education and smoking behavior. We extracted medical record data from six medical examination stations across the country of all Austrian military conscripts (aged 17-19 years) recruited between 1983 and 2017 ( $n=1.5$ million). Weight and height were measured to calculate body mass index (BMI). Mean BMI increased from 22.7 to $24.3 \mathrm{~kg} / \mathrm{m}^{2}$ between 1983 and 2017 . Over time, the prevalence of obesity (BMI $\geq 30 \mathrm{~kg} / \mathrm{m}^{2}$ ) increased from $1.6 \%$ (95\% CI $1.6-1.7 \%$ ) to $8.2 \%$ (95\% CI $8.1-8.3 \%$ ). The prevalence of obesity among Austrian young men increased remarkably in the past 35 years. Higher levels of education appeared to be associated with lower prevalence of obesity, particularly among the non-smokers.
\end{abstract}

Keywords Population based - Time trend . Education · Smoking · Body mass index

\footnotetext{
L. Yang, $\mathrm{PhD}$

Department of Cancer Epidemiology and Prevention Research, Alberta Health Services, Holy Cross Centre, 2210-2nd Street SW, ACB, T2S 3C3 Calgary, AB, Canada

Preventive Oncology \& Community Health Sciences, Cumming School of Medicine, University of Calgary, Hospital Drive NW, Calgary, Alberta, Canada
}

L. Yang, PhD · Univ.-Prof. T. Waldhoer, $\mathrm{PhD}(\varangle)$

Department of Epidemiology, Center for Public Health,

Medical University of Vienna, Kinderspitalgasse 15, Vienna, Austria

thomas.waldhoer@meduniwien.ac.at

A. Juan, MA

Ministry of Defence, Vienna, Austria

\section{Introduction}

The contemporary global disease burden is dominated by chronic diseases [1]. The management of chronic diseases is complex given that multiple risk factors may contribute to the development of these diseases. Nevertheless, a number of highly prevalent chronic diseases have excess adiposity as an established cause: type 2 diabetes mellitus, [2, 3] coronary heart disease, [4, 5] high blood pressure, [6, 7] high blood cholesterol, [8] and some cancers [9]. In particular, excess obesity during adolescence and young adulthood increases the risk of obesity [10] and risk of chronic diseases later in adulthood [11, 12].

The most common measure of weight status used to monitor population-wide obesity is the body mass index (BMI), based on which global obesity has nearly tripled since 1975 [13]. The prevalence of overweight and obesity is steadily increasing worldwide, with strong socioeconomic inequalities observed in many developed countries [13].

A number of previous studies reported the weight status in Austria, [14-20] limited to the use of selfreported data, conducted at least a decade ago, and none considered smoking, an important factor influencing obesity. The present study aims to quantify the time trend in weight status in young men over three and half decades using the health examination data from Austrian conscripts. Secondary aim was to explore if the time trend in weight status differs by education and smoking behavior to inform medical practice and public health policy. 


\section{Methods}

\section{Study population}

All Austrian men who reach the age of 18 years in a given year are enlisted in that year for either compulsory military or alternative service. The enlistment is preceded by an obligatory medical examination to verify military fitness. Details of this medical examination have been provided elsewhere [21-25]. In brief, the data set contains anonymized data of all young men in Austria in the year they reached age 18 years, including all years from 1983 through 2017. Therefore, this data set represents the total male population. Conscripts between the age of 17 and 19 years at the time of medical investigation were included for the present analyses (8\% excluded). We included young men who have complete data on education, weight and height and provinces of residence from the 1983 conscript cohort to the 2017 conscript cohort $(9.6 \%$ missing). The study design and conduct of the research obtained approval from the Medical University of Vienna Ethics Review Board (ECS 1393/2018).

\section{Weight status}

A total number of six stations in Austria facilitate conscription medical examination, covering 9 provinces: Burgenland + Vienna, Carinthia + Salzburg, Tyrol + Vorarlberg, Styria, Upper Austria and Lower Austria. These routinely collected data are stored in the electronic system of the medical examination stations and are centralized at the Austrian Ministry of Defense.

Height and weight were measured following standard procedures by trained technicians using standardized equipment, with Austrian conscripts wearing underwear and no shoes. Height was measured using a standard anthropometer, and body weight was determined to the nearest $100 \mathrm{~g}$ on calibrated scales $[21,24,26]$. The BMI was calculated as weight in $\mathrm{kg} /$ (height in meters) $^{2}$. The age range of the Austrian conscripts is around the upper limit of the US Centers for Disease Control and Prevention growth chart, which provides each BMI with a corresponding BMI-for-age percentile (2-19 years) [27]. This approach uses the 5th, 85th and 95th percentiles to define weight category: below 5 th percentile (underweight); 5 th $-<85$ th percentiles (normal weight); 85 th $-<95$ th percentile (overweight) and $\geq 95$ th (obese). In our study sample, the corresponding BMI for these percentiles were 18.3, 26.0, and $30.0 \mathrm{~kg} / \mathrm{m}^{2}$, which approximate to the standard BMI categories cut-off in adults ( $\geq 20$ years). Therefore, we categorized study participants into standard BMI categories defined by the world health organization: underweight $\left(<18.5 \mathrm{~kg} / \mathrm{m}^{2}\right)$, normal weight $\left(18.5-<25 \mathrm{~kg} / \mathrm{m}^{2}\right)$, overweight $\left(25-<30 \mathrm{~kg} / \mathrm{m}^{2}\right)$, class I obesity $\left(\geq 30.0-<35 \mathrm{~kg} / \mathrm{m}^{2}\right)$, class II obesity $\left(\geq 35.0-<40 \mathrm{~kg} / \mathrm{m}^{2}\right)$, and class III obesity $\left(\geq 40 \mathrm{~kg} / \mathrm{m}^{2}\right)$ [28].

\section{Individual and social factors}

We retrieved data of individual characteristics including smoking and education. Smoking was self-reported with yes/no responses to the question "do you currently smoke?" and classified into smokers (yes) and non-smokers (no). Data on smoking were available between 1991 and 2017. Education was classified into four categories: low (fewer than 9 years of compulsory school), medium (completed compulsory school), high (graduated from professional training or served an apprenticeship) and very high (qualified for university entrance), then collapsed to two groups: low to medium, and high to very high.

In total, there are 121 districts in Austria. Among the six stations in Austria which facilitate conscription medical examination covering nine provinces, Vienna is the most urban area with 3738 persons per $\mathrm{km}^{2}$ and a total of 1.8 million inhabitants, while population densities in other provinces (Burgenland, Carinthia, Lower Austria, Upper Austria, Salzburg, Styria, Tyrol, and Vorarlberg) are less than 2500 persons per $\mathrm{km}^{2}$ [23].

\section{Statistical analyses}

We grouped years of conscription by 5 -year intervals in 1 wave ( 7 waves over 35 years) to provide stable figures for BMI subtypes with small counts. Descriptive statistics were analyzed in the most recent wave of conscript cohort (2013-2017) to present the contemporary status of education level, smoking behavior, and the 5th, 50th and 95th percentiles of BMI in each stratum of included factors.

To explore time trends in weight status from conscript cohort 1983-1987 to 2013-2017, we calculated the crude prevalence of class I-III and class III obesity in each conscript cohort by regions. Further, crude and multivariable adjusted logistic regression were used to evaluate time trend in classes I-III and class III obesity using conscript cohort as a predictor.

Next, we calculated the crude prevalence of obesity classes I-III and obesity class III in each conscript cohort (1991-1992 as reference group, 1993-1997 to 2013-2017) by subgroups defined by education and smoking (data on smoking are available from 1991 onwards). To explore the effect of education and smoking on the prevalence of obesity, multivariable adjusted logistic regressions were calculated including conscript cohort, age, smoking and education as independent and prevalence as dependent variable.

We tested for interaction effects between conscript cohort and education as well as smoking, respectively. Significant interactions were found for both education and smoking; therefore, the multivariable logistic regression was further grouped by level of education 
and smoking. Statistical significance for testing interaction and trends of Odds Ratio (OR) was set at $P<0.05$. $P$-values were not adjusted for multiple tests and should be interpreted explanatorily only. All analyses were performed using SAS version 9.4 (SAS Institute Inc., Cary, NC, USA).

\section{Results}

Data on 1,507,040 young men were used for analyses. Over 1.5 million young men were grouped by 5 -year interval in 7 conscript cohorts, with the minimum size of 192,669 young men in each cohort. Among conscript cohort 2013-2017, obesity was prevalent $(8.2 \%$, 95\% CI 8.1-8.3\%) (Table 1). Majority of the conscript cohort had normal weight $(66.2 \%, 95 \%$ CI $66.0-66.4 \%)$, did not smoke (56.4\%) and had low to medium education $(72.4 \%)$. The overall prevalence of obesity increased from $1.6 \%$ (95\% CI: $1.6-1.7 \%$ ) to $8.2 \%(95 \%$ CI: $8.1-8.3 \%)$ over 35 years. A more than 10 -fold increase in prevalence from $0.04 \%$ to $0.5 \%$ was observed in obesity class III (Table 1).

In support, Fig. 1 illustrates that the most significant increases were observed in the higher BMI percentiles among Austrian conscripts from 1983 to 2017.

In the logistic regression adjusting for year of birth and age only, class I-III obesity exhibited a significant increase from 1983-1987 to 2013-2017 ( $p$-value for trends <0.001). We observed consistent association between education level and smoking status with obesity prevalence across conscript cohort similar to Fig. 2. Owing to the significant interaction between conscript cohort, education and smoking, we presented the estimated ORs and 95\% CI of conscript

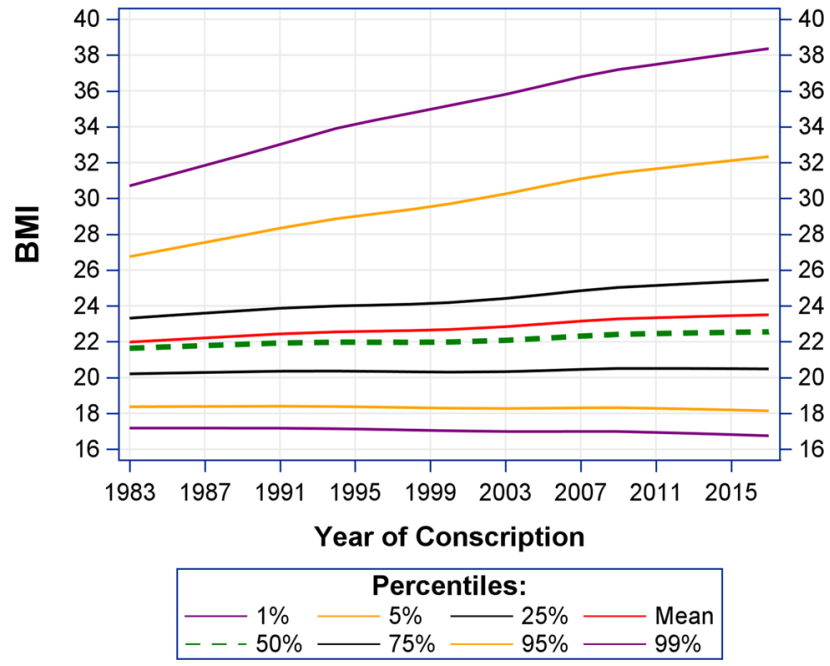

Fig. 1 Time trends in BMl by percentiles among Austrian conscript between 1983 and 2017

cohort by education and smoking status, adjusting for age from 1991 to 2017 (Table 2 and Fig. 2). Across all stations, we observed increased prevalence of obesity in both education groups (all $p$-values for trend $<0.001)$.

\section{Discussion}

In a population-based sample of young men, the prevalence of obesity has been rapidly increasing over the last three and half decades in Austria, suggesting an urgent need of preventive measures to curb this trend. By 2017, only $64.9 \%$ young men in Austria

Table 1 Characteristics of Austrian conscript cohorts between 1983 and 2017 (aged 17-19 years)

\begin{tabular}{|c|c|c|c|c|c|c|c|}
\hline & \multirow[b]{2}{*}{$N$} & \multicolumn{2}{|c|}{ Underweight $(\mathrm{BMI}<18.5)$} & \multicolumn{2}{|c|}{ Overall obese I-III (BMI $\geq 30$ ) } & \multicolumn{2}{|c|}{ Obesity class III (BMI $\geq 40)$} \\
\hline & & Prevalence $\%$ & $95 \% \mathrm{Cl}$ & Prevalence \% & $95 \% \mathrm{Cl}$ & Prevalence $\%$ & $95 \% \mathrm{Cl}$ \\
\hline Total & 1503740 & 5.98 & $5.94-6.02$ & 4.80 & $4.76-4.83$ & 0.24 & $0.23-0.25$ \\
\hline \multicolumn{8}{|l|}{ Age (years) } \\
\hline 17 & 764889 & 6.60 & $6.55-6.66$ & 4.48 & $4.43-4.52$ & 0.22 & $0.21-0.23$ \\
\hline 18 & 738851 & 5.32 & $5.28-5.38$ & 5.13 & $5.08-5.18$ & 0.27 & $0.25-0.28$ \\
\hline \multicolumn{8}{|l|}{ Education } \\
\hline Low to medium & 1089107 & 5.79 & $5.75-5.84$ & 5.50 & $5.45-5.54$ & 0.29 & $0.28-0.30$ \\
\hline High to very high & 410755 & 6.42 & $6.35-6.50$ & 2.96 & $2.91-3.01$ & 0.12 & $0.11-0.13$ \\
\hline \multicolumn{8}{|l|}{ Smoking } \\
\hline No & 623344 & 6.32 & $6.26-6.38$ & 4.86 & $4.80-4.91$ & 0.26 & $0.25-0.28$ \\
\hline Yes & 482618 & 5.78 & $5.72-5.85$ & 7.07 & $6.99-7.14$ & 0.38 & $0.36-0.40$ \\
\hline \multicolumn{8}{|l|}{ Cohort } \\
\hline 1983-1987 & 258294 & 5.81 & $5.72-5.90$ & 1.63 & $1.58-1.68$ & 0.04 & $0.03-0.05$ \\
\hline 1988-1992 & 221681 & 5.50 & $5.40-5.59$ & 2.65 & $2.59-2.72$ & 0.07 & $0.06-0.08$ \\
\hline 1993-1997 & 192823 & 5.57 & $5.47-5.67$ & 3.88 & $3.79-3.97$ & 0.15 & $0.14-0.17$ \\
\hline 1998-2002 & 206855 & 6.37 & $6.26-6.47$ & 4.36 & $4.27-4.45$ & 0.21 & $0.19-0.23$ \\
\hline 2003-2007 & 212456 & 6.21 & $6.11-6.31$ & 6.26 & $6.16-6.36$ & 0.31 & $0.28-0.33$ \\
\hline 2008-2012 & 218962 & 5.71 & $5.62-5.81$ & 7.52 & $7.40-7.63$ & 0.46 & $0.43-0.48$ \\
\hline 2013-2017 & 192669 & 6.77 & $6.66-6.88$ & 8.18 & $8.06-8.30$ & 0.54 & $0.51-0.58$ \\
\hline
\end{tabular}


Table 2 Multivariable adjusted model for class I-III obesity by education and smoking status

\begin{tabular}{|c|c|c|c|c|}
\hline & \multicolumn{2}{|c|}{ Low to median education } & \multicolumn{2}{|c|}{ High to very high education } \\
\hline & Non-smokers & Smokers & Non-smokers & Smokers \\
\hline & OR (95\% Cl) & OR $(95 \% \mathrm{Cl})$ & OR (95\% Cl) & OR $(95 \% \mathrm{Cl})$ \\
\hline \multicolumn{5}{|l|}{ Age (years) } \\
\hline 17 & 1 & 1 & 1 & 1 \\
\hline 18 & 1.20 (1.16 to 1.24$)$ & 1.14 (1.10 to 1.17$)$ & 1.45 (1.37 to 1.54$)$ & 1.39 (1.28 to 1.51$)$ \\
\hline \multicolumn{5}{|l|}{ Cohort } \\
\hline 1991-1992 & 1 & 1 & 1 & 1 \\
\hline 1993-1997 & $1.23(1.14-1.32)$ & $1.25(1.16-1.34)$ & $1.28(1.12-1.46)$ & $1.36(1.12-1.66)$ \\
\hline 1998-2002 & $1.34(1.25-1.43)$ & $1.28(1.19-1.38)$ & $1.60(1.39-1.83)$ & $1.55(1.27-1.89)$ \\
\hline 2003-2007 & $2.09(1.95-2.23)$ & $1.99(1.86-2.13)$ & $2.16(1.90-2.45)$ & $2.22(1.83-2.69)$ \\
\hline 2008-2012 & $2.59(2.44-2.77)$ & $2.61(2.44-2.79)$ & $2.42(2.14-2.74)$ & $2.73(2.25-3.31)$ \\
\hline 2013-2017 & $3.10(2.91-3.31)$ & $2.94(2.74-3.15)$ & $2.77(2.45-3.13)$ & $3.09(2.55-3.76)$ \\
\hline
\end{tabular}

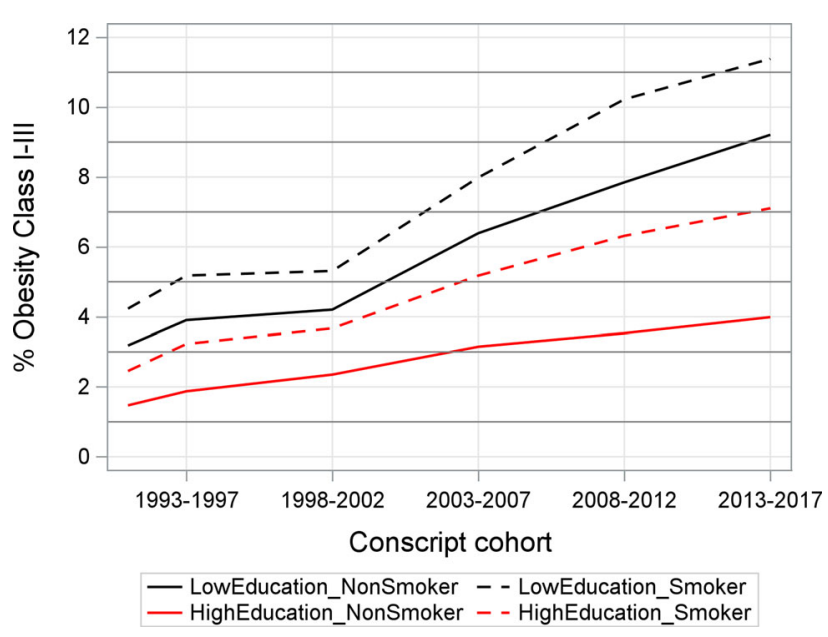

Fig. 2 Trends in obesity class I-III by education and smoking status

were with normal weight. The prevalence of overweight and obesity increased in the overall Austrian young men, and in each subgroup defined by education and smoking status. Nevertheless, the prevalence of obesity is constantly higher among those with low vs. high education level, and smokers vs. nonsmokers. Importantly, the difference in prevalence of obesity between education levels and smoking status appeared to widen over time.

Excessive weight and the simultaneous increase in obesity-related chronic conditions lead to premature mortality, morbidity, and long-term disability [29, 30]. Hence, a significant proportion of healthcare, economical, and societal burdens associated with chronic diseases could be potentially reduced by managing weight status. The 2015 World Health Organization Commission reported that adolescence can be a critical time or excess weight gain [31].

In the present study, we report rapidly increasing obesity in young men aged $17-<19$ years in Austria over the past 35 years. A previous study using five Aus- trian nationally representative cross-sectional survey data reported an increasing trend in adults BMI and prevalence of obesity (BMI $>30 \mathrm{~kg} / \mathrm{m}^{2}$ ) from 1973 to 2007, particularly among the low educational groups, [17] and men in western Austria [18]. Another survey study was conducted among Austrian farmers in $1999 / 2000$, reporting the prevalence of obesity peaked at 15-19 years old, and the eastern areas of Austria [16]. Although both studies were limited by using self-reported measures, another two Austria-based studies reported routinely collected national data on objectively measured weight and height among male conscripts. Based on the most recent study, the relevance of overweight and obesity among Austrian young men were $20.57 \%$ and $8.36 \%$, respectively, in 2010 [15]. Both conscript data-based studies found an increasing gradient of higher BMI from west to east of Austria [14]. Although these data are striking, none of the previous studies considered smoking and its interaction with education.

We observed socioeconomic inequalities, similar to those reported in other developed countries. For instance, lower level of education or urbanization appeared to be associated with larger body size in US children and adolescents [32] and adults [33]. Whereas for the UK children and adolescents, the observed BMI socioeconomic inequalities were historically small but widened overtime [34]. Notably, our data suggested a widening gap in obesity prevalence across sociodemographic groups defined by education level and smoking status.

In this study only smoking and education have been included which may be seen as a potential limitation. Both variables are good proxies for sociodemographics thereby adjusting for other effects like alcohol consumption and physical activity to some extent. Furthermore, data on alcohol consumption and physical activity were not collected and therefore we were not able to include those variables. Still, we think that smoking and education status are able to 
provide an unbiased estimate for the prevalence of obesity.

In conclusion, the prevalence of obesity has been rapidly increasing in Austrian young men. Higher level of education appeared to be associated with lower prevalence of obesity, particularly among the nonsmokers. Nevertheless, medical practice and public health policy should join forces in promoting the awareness of obesity in youth, and design populationlevel obesity prevention strategies and target interventions for the high-risk groups to reduce weight gaps within the population.

Author contribution Protocol/project development: LY, TW.Data collection or management: AJ, TW. Data analysis: LY, TW. Manuscript writing/editing: LY, AJ, TW

Funding This research did not receive any specific grant from funding agencies in the public, commercial, or not-for-profit sectors.

Funding Open access funding provided by Medical University of Vienna.

\section{Declarations}

Conflict of interest L. Yang, A. Juan and T. Waldhoer declare that they have no competing interests.

Ethical standards For this article no studies with human participants or animals were performed by any of the authors. All studies performed were in accordance with the ethical standards indicated in each case. This retrospective study was performed after consultation with the institutional ethics committee and in accordance with national legal requirements. No informed consent was needed as analysis was based on anonymized data only. Ethical approval was obtained (ECS 1393/2018). Data were anonymized as well as retrospective.

Open Access This article is licensed under a Creative Commons Attribution 4.0 International License, which permits use, sharing, adaptation, distribution and reproduction in any medium or format, as long as you give appropriate credit to the original author(s) and the source, provide a link to the Creative Commons licence, and indicate if changes were made. The images or other third party material in this article are included in the article's Creative Commons licence, unless indicated otherwise in a credit line to the material. If material is not included in the article's Creative Commons licence and your intended use is not permitted by statutory regulation or exceeds the permitted use, you will need to obtain permission directly from the copyright holder. To view a copy of this licence, visit http://creativecommons.org/licenses/by/4.0/.

\section{References}

1. Lim SS, Vos T, Flaxman AD, et al. A comparative risk assessment of burden of disease and injury attributable to 67 risk factors and risk factor clusters in 21 regions, 1990-2010: a systematic analysis for the Global Burden of Disease Study 2010. Lancet. 2012;380:2224-60.

2. Colditz GA, Willett WC, RotnitzkyA, Manson JE. Weight gain as a risk factor for clinical diabetes mellitus in women. Ann Intern Med. 1995;122:481-6.
3. Vazquez G, Duval S, Jacobs DR Jr, Silventoinen K. Comparison of body mass index, waist circumference, and waist/hip ratio in predicting incident diabetes: a meta-analysis. Epidemiol Rev. 2007;29:115-28.

4. Canoy D. Distribution of body fat and risk of coronary heart disease in men and women. Curr Opin Cardiol. 2008;23:591-8.

5. Global Burden of Metabolic Risk Factors for Chronic Diseases Collaboration (BMI Mediated Effects). Metabolic mediators of the effects of body-mass index, overweight, and obesity on coronary heart disease and stroke: a pooled analysis of 97 prospective cohorts with 1.8 million participants. Lancet. 2014;383(9921):970-83.

6. Kristjansson K, Sigurdsson JA, Lissner L, Sundh V, Bengtsson C. Blood pressure and pulse pressure development in a population sample of women with special reference to basal body mass and distribution of body fat and their changes during 24 years. Int J Obes Relat Metab Disord. 2003;27:128-33.

7. Droyvold WB, Midthjell K, Nilsen TI, Holmen J. Change in body mass index and its impact on blood pressure: a prospective population study. Int JObes. 2005;29:650-5.

8. Gostynski M, Gutzwiller F, Kuulasmaa K, et al. Analysis of the relationship between total cholesterol, age, body mass index among males and females in the WHO MONICA Project. IntJ Obes Relat Metab Disord. 2004;28:1082-90.

9. Kyrgiou M, Kalliala I, Markozannes G, et al. Adiposity and cancer at major anatomical sites: umbrella review of the literature. BMJ. 2017;356:j477.

10. Guo SS, Wu W, Chumlea WC, Roche AF. Predicting overweight and obesity in adulthood from body mass index values in childhood and adolescence. Am J Clin Nutr. 2002;76:653-8.

11. Ohlsson C, Bygdell M, Sondén A, Jern C, Rosengren A, Kindblom JM. BMI increase through puberty and adolescence is associated with risk of adult stroke. Neurology. 2017;89:363-9.

12. Skinner AC, Perrin EM, Moss LA, Skelton JA. Cardiometabolic risks and severity of obesity in children and young adults. NEngl J Med. 2015;373:1307-17.

13. The Lancet Public Health. Tackling obesity seriously: the time has come. Lancet Public Health. 2018;3:e153.

14. Kirchengast S, Schober E, Waldhör T, Sefranek R. Regional and social differences in body mass index, and the prevalence of overweight and obesity among 18 year old men in Austria between the years 1985 and 2000. Coll Antropol. 2004;28:541-52.

15. Poglitsch M, Kefurt R, Mittlböck M, et al. Prevalence of obesity and overweight in male 18-year-olds in Austria from 2006 to 2010: an update. Eur Surg. 2011;43:181-6.

16. Dorner T, Leitner B, Stadlmann H, et al. Prevalence of overweight and obesity in Austrian male and female farmers. SozPraventivmed. 2004;49:243-6.

17. Großschädl F, Stronegger WJ. Long-term trends in obesity among Austrian adults and its relation with the social gradient: 1973-2007. Eur J Public Health. 2012;23:306-12.

18. GroßschädlF, Stronegger WJ. Regional trends in obesity and overweight among Austrian adults between 1973 and 2007. Wien Klin Wochenschr. 2012;124:363-9.

19. Borena W, Stocks T, Strohmaier S, et al. Long-term temporal trends in cardiovascular and metabolic risk factors. Wien Klin Wochenschr. 2009;121:623.

20. Ulmer H, Kelleher CC, Fitz-Simon N, Diem G, Concin H. Secular trends in cardiovascular risk factors: an age-period cohort analysis of 698,954 health examinations in 181,350 Austrian men and women. J Intern Med. 2007;261:566-76. 
21. Hermanussen M, Danker-Hopfe H, Weber GW. Body weight and the shape of the natural distribution of weight, in very large samples of German, Austrian and Norwegian conscripts. Int JObes Relat Metab Disord. 2001;25:1550-3.

22. Kapusta ND, Pietschnig J, Plener PL, Bluml V, Lesch OM, Walter H. Does breath carbon monoxide measure nicotine dependence? J AddictDis. 2010;29:493-9.

23. Kapusta ND, Zorman A, Etzersdorfer E, Ponocny-Seliger E, Jandl-Jager E, Sonneck G. Rural-urban differences in Austrian suicides. Soc Psychiat Epidemiol. 2008;43:311-8.

24. Wallner A, Hirz A, Schober E, Harbich H, Waldhoer T. Evolution of cardiovascular risk factors among 18-yearold males in Austria between 1986 and 2005. Wien Klin Wochenschr. 2010;122:152-8.

25. Yang L, Vass C, Smith L, Juan A, Waldhor T. Thirty-five-year trend in the prevalence of refractive error in Austrian conscripts based on 1.5 million participants. Br J Ophthalmol. 2020;104(10):1338-44.

26. Kirchengast S, Schober E, Waldhor T, Sefranek R. Regional and social differences in body mass index, and the prevalence of overweight and obesity among 18 year old men in Austria between the years 1985 and 2000. Coll Antropol. 2004;28:541-52.

27. WHO Multicentre Growth Reference Study Group. WHO child growth standards: length/height-for-age, weight-forage, weight-for-length, weight-for-height and body mass index-for-age: methods and development. Geneva: World Health Organization; 2006.
28. James PT, Leach R, Kalamara E, Shayeghi M. The worldwide obesity epidemic. Obes Res. 2001;9(S11):228S-33S.

29. Kushner RF, Foster GD. Obesity and quality of life. Nutrition. 2000;16:947-52.

30. Visscher TL, Seidell JC. The public health impact of obesity. Annu Rev Public Health. 2001;22:355-75.

31. World Health Organization. Consideration of the evidence on childhood obesity for the commission on ending childhood obesity: report of the ad hoc working group on science and evidence for ending childhood obesity. 2016. https://www.who.int/news-room/fact-sheets/ detail/obesity-and-overweight. Accessed 23 Feb 2020.

32. Ogden CL, Fryar CD, Hales CM, Carroll MD, Aoki Y, Freedman DS. Differences in obesity prevalence by demographics and urbanization in US children and adolescents, 2013-2016. JAMA. 2018;319:2410-8.

33. Flegal KM, Kruszon-Moran D, Carroll MD, Fryar CD, Ogden CL. Trends in obesity among adults in the United States, 2005 to 2014. JAMA. 2016;315:2284-91.

34. Bann D, Johnson W, Li L, Kuh D, Hardy R. Socioeconomic inequalities in childhood and adolescent body-mass index, weight, and height from 1953 to 2015: an analysis of four longitudinal, observational, British birth cohort studies. Lancet Public Health. 2018;3:e194-e203.

Publisher's Note Springer Nature remains neutral with regard to jurisdictional claims in published maps and institutional affiliations. 